\title{
Hierarchical Structural Health Monitoring of Wind Turbines based on an Overview of Mechanical Damage
}

\author{
Norio Takeda ${ }^{1}$, and Tatsuya Kameyama ${ }^{2}$ \\ ${ }^{1}$ Center for Technology Innovation -Mechanical Engineering, Hitachi Ltd., Hitachinaka, Ibaraki, 312-0034, Japan \\ norio.takeda.uf@hitachi.com \\ ${ }^{2}$ Center for Technology Innovation -Artificial Intelligence, Hitachi Ltd., Kokubunji, Tokyo, 185-8601, Japan \\ tatsuya.kameyama.sd@hitachi.com
}

\begin{abstract}
A metric of giving an overview of mechanical damage has been proposed to practically realize the structural health monitoring of mechanical system using big data. In the case that a mechanical system includes many local hot spots, such as welded and bolt joints, where structural health should be monitored, the health monitoring system for all the spots could be a large and expensive one. To overcome this drawback and increase the number of machines under structural health monitoring, the concept of a hierarchical monitoring was applied to the structural health monitoring of wind turbines in this study. In the first stage of the hierarchical monitoring, an overview of mechanical damage is given using the proposed metric, and accordingly, target machines are extracted for additional evaluation. The local damage can be accurately evaluated at the hot spots in the additional evaluation, namely, the second stage of the hierarchical monitoring. As the result of employing the metric of giving the overview for several wind turbines, the extracted turbines corresponded to the turbines that are operated under severe wind conditions and expected for the accumulation of mechanical damage. It can be therefore concluded that the concept of the hierarchical monitoring is effective to the expansion of the monitoring target.
\end{abstract}

\section{INTRODUCTION}

The technologies on the prognostics and health management (PHM) of machines, such as wind turbines and trains, have been developed aggressively for last two decades. The technologies cover various topics: data acquisition, signal processing and modeling, and operation and maintenance (O\&M) practice. Although all these topics are important to realize an effective PHM, the modeling techniques are crucial

Norio Takeda et al. This is an open-access article distributed under the terms of the Creative Commons Attribution 3.0 United States License, which permits unrestricted use, distribution, and reproduction in any medium, provided the original author and source are credited. to evaluate the current and future health state of the machines. The modeling techniques are classified into two techniques, i.e., data-driven modeling and physics-based modeling (for example, Alemayehu, \& Ekwaro-Osire, 2017). As for the data-driven modeling, there are many contributions based on conventional survival analysis, Maharanobis-Taguchi system, neural networks, support vector machines, etc. (for example, Hasegawa, Ogata, Murakawa, Kobayashi, \& Ogawa, 2017; Asgarpour \& Sorensen, 2018). These data-driven techniques usually use the fault history data with or without the normal condition data of the machines, and some simple techniques have already been utilized in the process of O\&M practices. As well as the data-driven techniques, the physics-based modeling techniques are widely proposed to evaluate the health state of the machines accurately (Zhao, Tian, \& and Zeng, 2013; Alemayehu, \& Ekwaro-Osire, 2015; Ewing, Thies, Shek, \& Bittencourt, 2019). In the case of the physicsbased modeling, it is a challenge to prepare a model of calculating mechanical responses accurately with low computational cost. Data assimilation is one of the techniques for improving the accuracy of the physical model, and hence, the data assimilation was applied to the physics-based modeling of wind turbines (Namura, Muto, Ueki, \& Takeda, 2020).

The physics-based modeling has the advantage of evaluating the state of the machines with fewer data compared to the data-driven modeling, and accordingly, it can be applied from the start of machine operation, when fault history does not exist. Nowadays the physics-based modeling is usually utilized to evaluate the structural health of a specific part or component included in the machines, such as gear, welded joint, bolt, bearing, gearbox, welded structure, etc. (for example, Soualhi, Hawwan, Medjaher, Clerc, Hubert, \& Guillet, 2018). On the other hand, it is expected for maintenance practitioners in industry to evaluate the structural health state not only for a specific part or component but also for all critical components and all the machines. They would like to determine which part or 
component to inspect and prioritize all machines in order of the health sate depending on the result of the evaluation.

To realize the evaluation of the structural health for all the parts and components in a whole machine, a hierarchical structural health monitoring is promising and therefore introduced in this study. In the first stage of the hierarchical health monitoring, an overview of mechanical damage is given to prioritize all the target machines and extract the machines to be investigated in the second stage according to a metric proposed. In addition to the concept of the hierarchical health monitoring, an illustrative system configuration and damage evaluation results are shown by addressing the structural health monitoring of wind turbines.

As for the condition monitoring of wind turbines, various kinds of technologies have already been proposed and applied in industrial practices (for example, Stetco, Dinmohammadi, Zhao, Robu, Flynn, Barnes, Keane, \& Nenadic, 2019; Reddy, Kumar, \& Raju, 2011). The proposed monitoring is unique in terms of monitoring all critical components with a physics-based model of whole wind turbine, i.e. the digital twin of wind turbine, and hierarchical monitoring concept.

\section{Hierarchical Structural Health Monitoring}

A hierarchical health monitoring is introduced to practically realize real-time evaluation of mechanical damage and decision making for maintenance. The hierarchical health monitoring is performed using a metric based on an overview of the mechanical damage. A hierarchical monitoring system has been constructed to confirm the effectiveness of the monitoring.

The applicability of this hierarchical health monitoring is limited in terms of failure mode and data required for the monitoring. It is well known that the failure rate of mechanical components changes according to the bathtub curve, which represents three different failures through operating life, i.e., early failures, random failures, and wearout failures. The proposed metric can be employed to compare the fatigue damage of the components between target machines, and therefore, this hierarchical monitoring is applicable to the monitoring of the wear-out failures. In addition, the loads applied to the components should be known to utilize the proposed monitoring because the proposed metric is evaluated with the applied load as derived in section 2.2. The easiest way to obtain the loads is the computational simulation of target machine, namely digital twin. In this case, the design information of target machine is required for the simulation as well as the data measured for the control and/or maintenance of machines, and hence, the manufacturer of target machine can easily apply the proposed monitoring.

\subsection{Concept of Hierarchical Structural Health Monitoring}

The fatigue damage of mechanical structures is generally evaluated at hot spots, such as welded parts, bolt joints and gears, according to Palmgren-Miner rule.

$$
D=\sum_{i} \frac{n_{i}}{N_{i}}
$$

where, $n_{i}$ is the number of stress amplitude $\sigma_{i}$ observed in a stress time history, and $N_{i}$ is the number of cycles to fatigue failure based on material database, for example, stress-strain curve of welded parts and bolts. The damage evaluation according to Eq. (1) is widely used, and the effectiveness of the evaluation is proven. It is, however, difficult to use Eq. (1) when monitoring the structural health of a whole machine, such as a wind turbine, a train and construction machinery. There are many hot spots where fatigue damage should be evaluated in a mechanical system. In the case of the wind turbine, Hitachi HTW2.0-80, the number of bolts connecting a blade to the hub of the turbine is more than 50 . The tower of the turbine is constructed using welded and bolt joints, and accordingly, there are 20 welded sections and the number of bolts arranged at a flange part of the tower ranges from 70 to 140. As a result, a monitoring system for evaluating the structural health, i.e., fatigue damage, at all the hot spots could be a large and expensive one. To overcome this drawback and increase the number of machines under the structural health monitoring, the concept of a hierarchical monitoring was applied to the monitoring of wind turbines in this development as shown in Figure 1.

In the first stage of the hierarchical monitoring, an overview of mechanical damage is evaluated for all machines to be monitored, and then target machines are extracted among all machines for additional and detailed damage evaluation. A metric is employed to give an overview of mechanical damage here. The damage metric plays an important role in the hierarchical monitoring, and therefore, it is introduced in the following section. The detail of mechanical damage can be accurately evaluated at the hot spots in the additional monitoring.

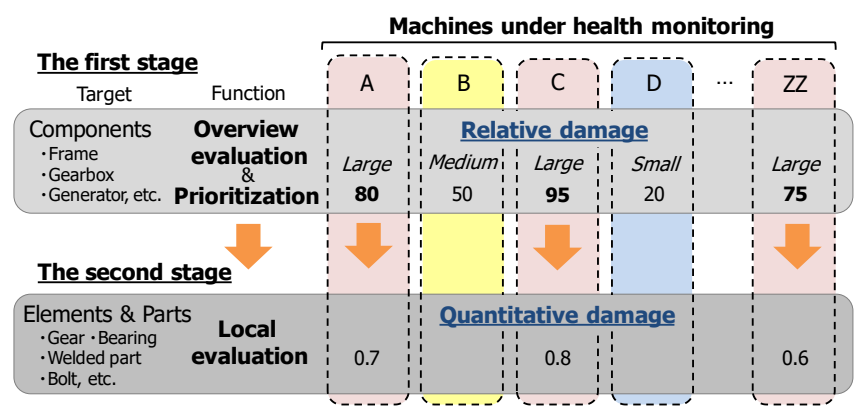

Figure 1. Concept of hierarchical structural health monitoring. 


\subsection{Structural Health Monitoring based on an Overview of Mechanical Damage}

A metric for giving an overview of mechanical damage is defined as

$$
D \text { factor }=\sum_{i} R_{i}^{m} \cdot n_{i}
$$

where, $R_{i}$ is the force or moment that occurs at a point on a component included in a machine, and $n_{i}$ is the number of load amplitude $R_{i}$ observed in a force or moment time history. The parameter $m$ is the slope of the stress-strain curve. The force and moment occurring at a point have six elements, i.e., three forces and three moments, and accordingly, six $D$ factors are calculated at a point. In the case that fatigue failure of a component largely depends on the forces and moments occurring at a point on the component, the risk of the fatigue failure can be estimated according to the $D$ factors. For example, the fatigue damage of the bolts connecting a blade to the hub of a wind turbine depends on the forces and moments that occurs at the root of the blade. The $D$ factors estimated from the forces and moments at the root of the blade give an overview of the fatigue damage of the bolts. Thus, the $D$ factors give an overview of fatigue damage without the local damage evaluation with Eq. (1). This skip of the local evaluation saves computational cost dramatically and makes structural health monitoring practical, e.g., the number of damage evaluations for the blade bolts is decreased from more than 50 to six.

The advantage of the damage evaluation with the $D$ factor is that the priority of maintenance is determined by comparing the $D$ factors among the machines under the health monitoring. On the other hand, local and quantitative damage evaluation cannot be performed with the overview, and therefore, the local and quantitative evaluation is executed for the machines that are extracted according to the $D$ factor.

\subsection{Illustrative System of Hierarchical Health Monitoring}

A trial system for monitoring the structural health of wind turbines is constructed to confirm the effectiveness of the proposed health monitoring. The configuration of the system is shown in Figure 2. The data of the wind turbines are collected every one second and 10 minutes with supervisory control and data acquisition (SCADA) system. The SCADA data with the sampling frequency of $1 \mathrm{~Hz}$ is employed to realize the proposed health monitoring, and hence, the $1 \mathrm{~Hz}$ data is accumulated to a data storage once. The data accumulated is then transferred to a high-performance computer on which the digital twins of wind turbines run to obtain the forces and moments that occur at several key points of wind turbines. Although the condition monitoring methods based on the SCADA data have already been proposed (for example, Yang, Court, \& Jiang, 2013; Pandit, \& Infield, 2018; Leahy, Hu, Konstantakopoulos, Spanos,
Agogino, \& O'Sullivan, 2018), the SCADA data is specifically utilized as the inputs of the digital twin in this study. The forces and moments obtained are transferred from the high-performance computer to a data server that executes two applications, i.e., a database software for keeping the forces and moments and a digital dashboard software. Furthermore, the damage evaluation as mentioned in the previous section runs on a couple of high-end PCs. The results of the damage evaluation can be checked by accessing the data server from client PCs.

\section{STRUCTURAl Health MONITORING OF WiND TURBINES}

The damage evaluation according to the $D$ factor was applied to several wind turbines, and the priority of maintenance was made depending on the evaluation results.

\subsection{Results of Structural Health Monitoring}

The damage evaluation introduced in the previous chapter was applied to five wind turbines; the type of the wind turbines is Hitachi HTW2.0-80, and the turbines are operated at three different sites as shown in Table 1. The forces and moments were calculated at four points, namely, blade root, the tip of low speed shaft, tower top and tower bottom, on a wind turbine by using the digital twin of them. As the digital twin, the aero-servo-elastic simulation of the turbine was carried out in the time domain with OpenFAST provided by the National Renewable Energy Laboratory in the U.S.A (NREL). In this study, the digital twins run for 10 minutes every 30 minutes on the system shown in Fig. 2 because the CPU time of the simulation should catch up with the real time. The $D$ factors were accordingly evaluated with the outputs of 10 minutes' simulation and accumulated every 30 minutes.

Figure 3 shows the average $D$ factors of the tip of low speed shaft over two months, i.e., from October 1 st to November 30th, 2019. The structural reliability of the gearbox in the drivetrain of wind turbines depends on the forces and bending moments applied to the tip of low speed shaft. In particular, the rotational torque and two bending moments are critical to the reliability of the gear box.

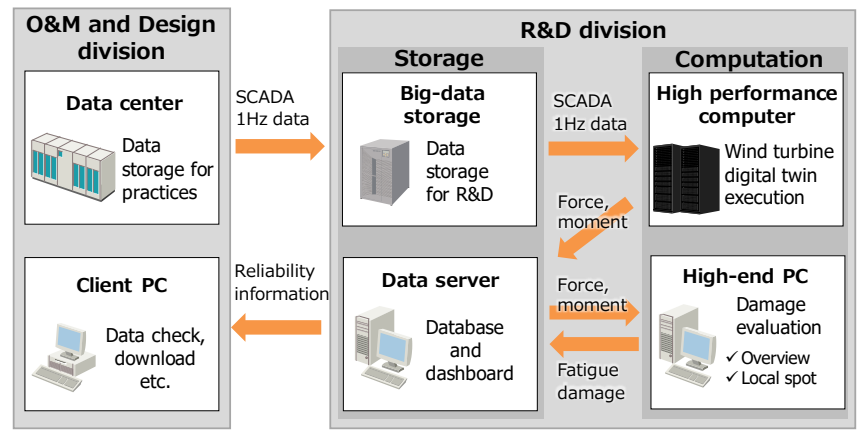

Figure 2. Configuration of hierarchical structural health monitoring system. 
The wind turbines listed in Table 1 can be therefore prioritized according to the $D$ factors for the torque and bending moments when planning the inspection of the gearbox. In the case of the $D$ factor for the bending moment around vertical axis, the $D$ factors of the site $\mathrm{C}$ are obviously larger than those of the site $\mathrm{A}$ and $\mathrm{B}$, even though there is not a certain tendency among the $D$ factors for the rotational torque. On the other hand, in the case of the $D$ factor for the bending moment around horizontal axis, the $D$ factor of the site A is obviously larger than those of the site B and C. As a result, it can be mentioned that the gearboxes of the wind turbines at site $\mathrm{A}$ and $\mathrm{C}$ had been more damaged than the gear box at the site $\mathrm{B}$ for the two months. When the fatigue lives of the turbine gearbox are different each other, there is a high possibility that the mechanical damage by the bending moment is one cause of the difference. In such case, failure history data are useful to determine which the $D$ factor to pay attention to.

In order to compare the mechanical damage of the gearbox during the lifetime of the wind turbines, the $D$ factors were accumulated from the start of operation to the end of December 2019 and shown in Figure 4. Although the $D$ factors for the bending moment around vertical axis at the site $\mathrm{C}$ were larger than that at the site A in Fig. 3, the lifetime $D$ factors of the site $\mathrm{A}$ are larger than those of the site $\mathrm{C}$ because of the long operation period for the site A. The accumulation of the $D$ factor should be therefore confirmed to make a decision on the extraction of the candidates to be analyzed in the second stage of the hierarchical structural health monitoring and the plan of maintenance.

In this example, the wind turbines at the site $\mathrm{A}$ and $\mathrm{C}$ should be extracted preferentially to evaluate the fatigue damage of the hot spots, i.e., gears, bearings, in the gearbox in the second stage because the lifetime $D$ factors of the site A is the largest among all the turbines and those of the site $\mathrm{C}$ is the second largest for the bending moment around vertical axis even though the shorter operation period of the site $\mathrm{C}$.

Table 1. Wind turbines monitored and start date of operation.

\begin{tabular}{c|c|c|c}
\hline No. & Site & WT number & $\begin{array}{c}\text { Start month and year of } \\
\text { operation }\end{array}$ \\
\hline 1 & A & 1 & March, 2012 \\
\hline 2 & B & 1 & March, 2014 \\
\hline 3 & & 1 & \multirow{2}{*}{ September, 2014 } \\
\cline { 1 - 1 } 4 & \multirow{2}{*}{ C } & 2 & \\
\cline { 3 - 3 } 5 & & 3 & \\
\cline { 3 - 3 } & & & \\
\hline
\end{tabular}

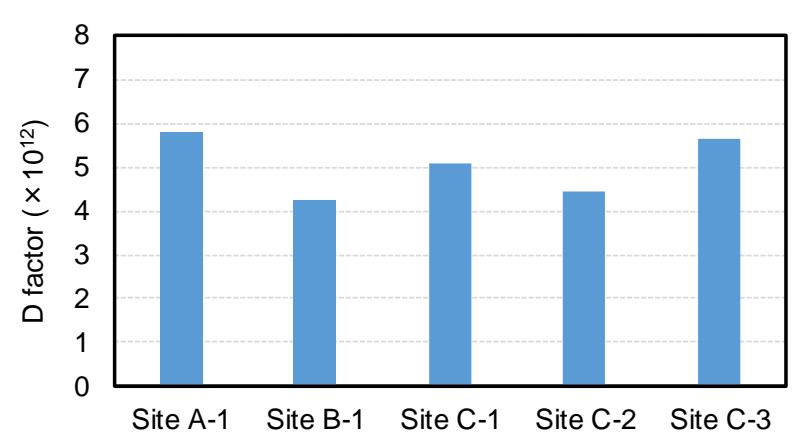

a) $D$ factors for rotational torque

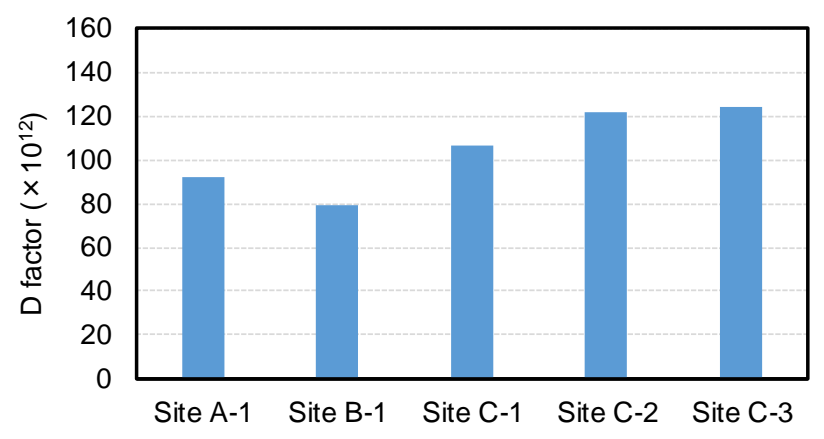

b) $D$ factors for bending moment around vertical axis

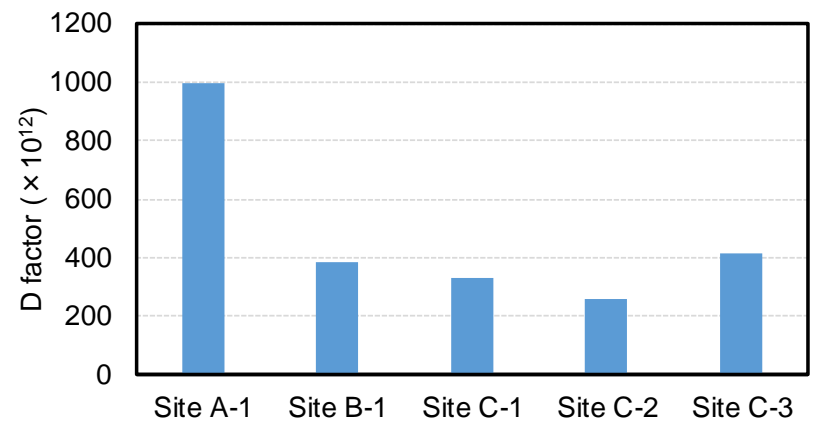

c) $D$ factors for bending moment around horizontal axis

Figure 3. Average $D$ factors evaluated at the tip of low speed shaft.

\subsection{Analysis of Environmental Root Cause}

Since the $D$ factor is directly derived from the forces or moments, it is easy to identify the environmental cause of a mechanical damage by investigating why the forces and/or moments become large for a wind turbine damaged. The 10 minutes mean and standard deviation of the wind speed and direction are summarized in Table 2. The mean and standard deviation were averaged over two months, namely, from October 1, 2019 to November 30, 2019. It can be noticed that the standard deviation of the wind direction at the site $\mathrm{C}$ is larger than the deviations at the site $\mathrm{A}$ and $\mathrm{B}$. 


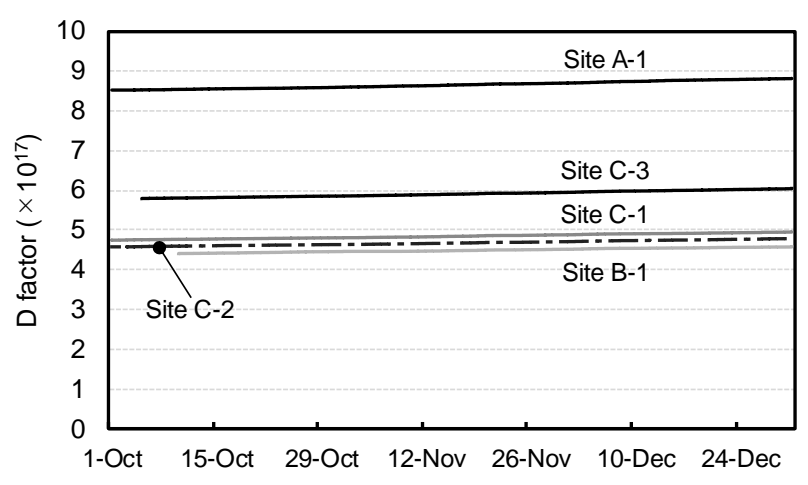

a) Accumulated $D$ factors for rotational torque

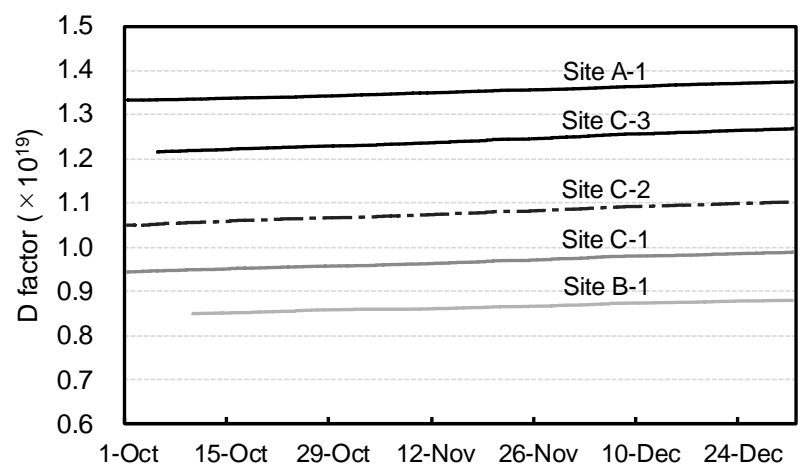

b) Accumulated $D$ factors for bending moment around vertical axis

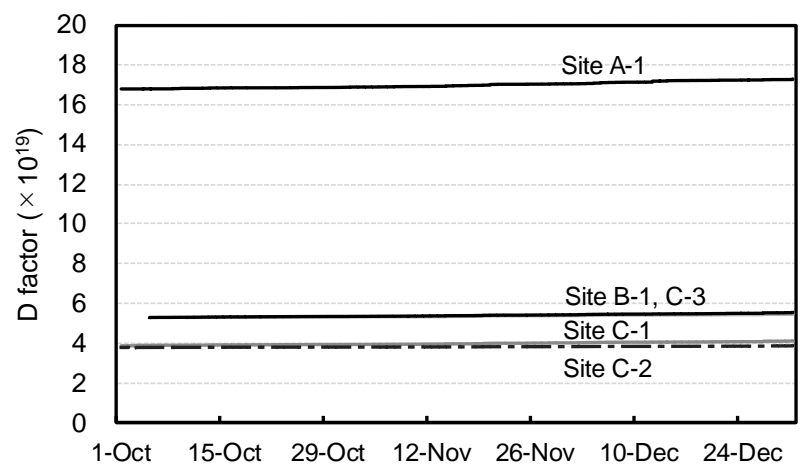

c) Accumulated $D$ factors for bending moment around horizontal axis

Figure 4. Accumulated $D$ factors related to the damage of the gearbox of wind turbine.

On the other hand, any specific tendency cannot be observed for the wind speed. To confirm these results visually, the time-series data of a wind turbine in the site $\mathrm{C}$ are compared with that in the site $\mathrm{A}$ for the wind speed and direction in Figure 5. It is visually obvious that the wind direction changes more frequently at the site $\mathrm{C}$. In addition to the timeseries data, the relation between the wind speed and direction is shown as wind rose for the wind turbines of the site A and
Table 2. 10 minutes mean and standard deviation of wind speed and direction averaged over two months.

\begin{tabular}{r|c|r|r|r|r}
\hline \multirow{2}{*}{ No. } & \multirow{2}{*}{$\begin{array}{c}\text { Site, WT } \\
\text { number }\end{array}$} & \multicolumn{2}{|c|}{$\begin{array}{c}\text { Wind speed } \\
{[\mathrm{m} / \mathrm{s}]}\end{array}$} & \multicolumn{2}{c}{$\begin{array}{c}\text { Wind direction } \\
\text { [deg.] }\end{array}$} \\
\cline { 3 - 6 } & & Mean & $\begin{array}{c}\text { Standard } \\
\text { deviation }\end{array}$ & Mean & $\begin{array}{c}\text { Standard } \\
\text { deviation }\end{array}$ \\
\hline 1 & A-1 & 6.68 & 0.80 & 204.8 & 22.1 \\
\hline 2 & B-1 & 6.29 & 0.63 & 225.4 & 23.5 \\
\hline 3 & C-1 & 5.91 & 0.92 & 217.4 & 81.2 \\
\hline 4 & C-2 & 5.66 & 0.98 & 139.9 & 89.9 \\
\hline 5 & C-3 & 6.36 & 0.96 & 217.2 & 84.0 \\
\hline
\end{tabular}
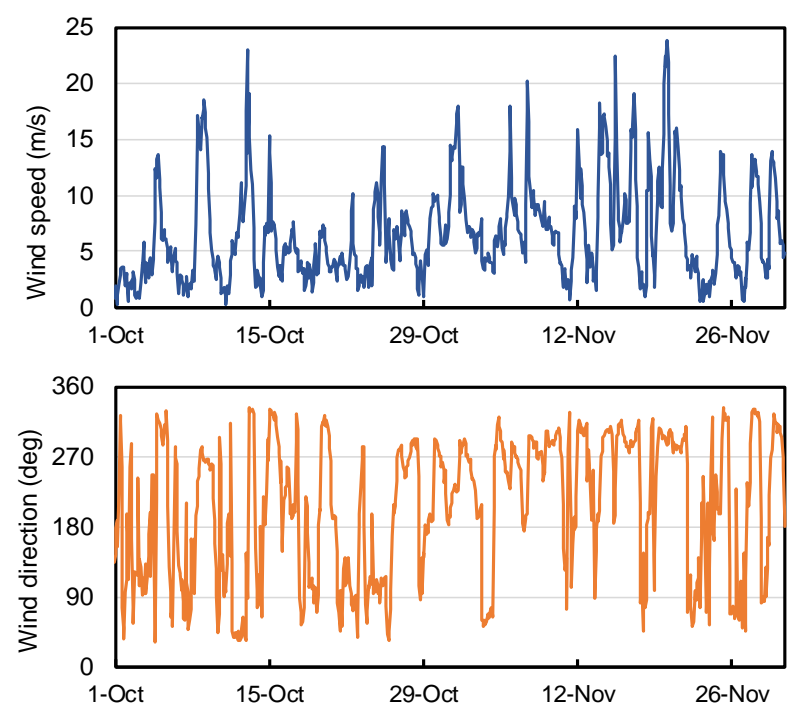

a) Wind conditions of the site A-1
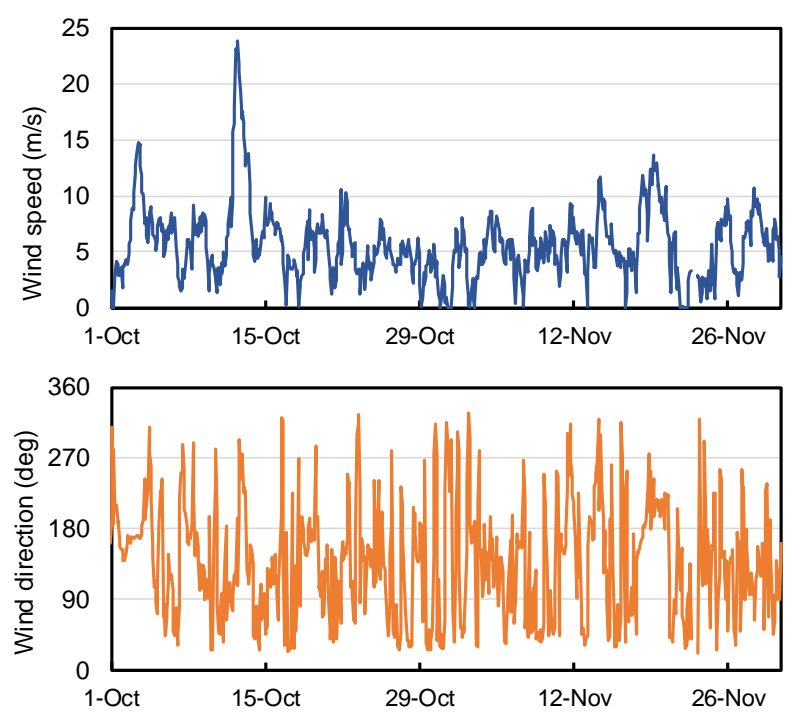

b) Wind conditions of the site $\mathrm{C}-2$

Figure 5. Time-series data for the wind conditions of the target wind turbines. 
$\mathrm{C}$ in Figure 6. The wind direction of the site $\mathrm{A}$ is distributed around west, but that of the site $\mathrm{C}$ is distributed over all directions.

In the case that the wind direction changes frequently, there are many opportunities for the large difference between the wind direction and the yaw direction of wind turbine. As the difference becomes large, the bending moment around vertical axis becomes large at the tip of the low speed shaft. It can be therefore concluded that the $D$ factor caused by the bending moment around vertical axis can be accumulated more rapidly because of the frequent change of the wind direction at the site $\mathrm{C}$.

\section{Conclusion}

A hierarchical health monitoring is proposed to realize a practical and real-time structural health monitoring of machines under operation. The fatigue damage of structures is conventionally evaluated at local hot spots, such as welded parts and bolts. There are huge hot spots in a whole machine, and consequently, it is not practical to construct a structural health monitoring system by applying the conventional damage evaluation. To overcome this drawback, a metric of giving an overview of mechanical damage was proposed and employed in the first step of the hierarchical health monitoring. An overview of mechanical damage is evaluated with the forces and bending moments that occur at a point on machine structure, and the forces and moments can be calculated by using the digital twin that simulates the behavior of machine.

An illustrative system of the hierarchical structural health monitoring was constructed for evaluating the fatigue damage of wind turbines. As the result of evaluating the damage using the metric proposed, the damage caused by the bending moment was accumulated more quickly at the tip of low speed shaft for three wind turbines included in a site.

In addition, the root cause of the damage was easily obtained by analyzing the relation between the bending moment and the wind condition of the site. It can be therefore concluded that the practical and real-time structural health monitoring is realized by the hierarchical health monitoring with the metric giving the overview of mechanical damage.

\section{REFERENCES}

Alemayehu, F. M., \& Ekwaro-Osire, S. (2015). Probabilistic performance of helical compound planetary system in wind turbine. Journal of Computational and Nonlinear Dynamics, vol. 10 (4), pages: 041003-12.

Alemayehu, F. M., \& Ekwaro-Osire, S. (2017). Probabilistic prognostics and health management: a brief summary, In Ekwaro-Osire, S., Goncalves, A. C., Alemayehu, F. M. (Eds.), Probabilistic Prognostics and Health Management of Energy Systems (3). Switzerland: Springer.

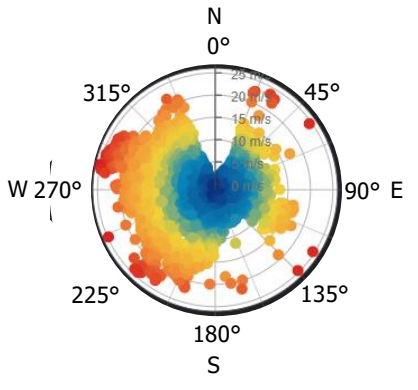

a) Site A-1

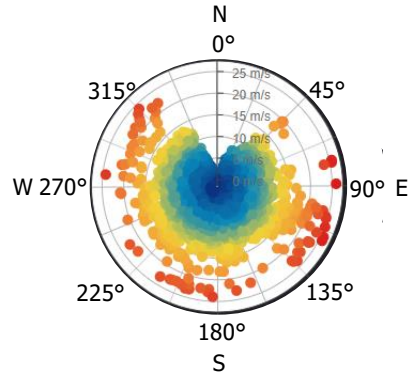

b) Site C-2
Figure 6. Relation between wind speed and direction.

Asgarpour, M., \& Sorensen, J. (2018). Bayesian based prognostic model for predictive maintenance of offshore wind farms. International Journal of Prognostics and Health Management, vol. 9 (1) 10, pages: 9.

Ewing, F., Thies, P. R., Shek, J. K., \& Bittencourt, C.Tian, Z., (2019). A physics-based prognostics approach for tidal turbines. IEEE Transactions on reliability 2019 IEEE International Conference on Prognostics and Health Management, June 17-19, CA, USA.

Hasegawa, T., Ogata, J., Murakawa, M., Kobayashi, T., \& Ogawa, T. (2017). Adaptive training of vibration-based anomaly detector for wind turbine condition monitoring. International Journal of Prognostics and Health Management, vol. 8 (2) 20, pages: 8.

Leahy, K., Hu, R. L., Konstantakopoulos, I. C., Spanos, C. J., Agogino, A. M., \& O’Sullivan, D. T. J. (2018). Diagnosing and predicting wind turbine faults from SCADA data using support vector machines. International Journal of Prognostics and Health Management, vol. 9 (1) 6, pages: 11.

Namura, N., Muto, K., Ueki, Y., \& Takeda, N. (2020). Model-based blade load monitoring of floating wind turbine enhanced by data assimilation. AIAA SciTech 2020 Forum, January 6-10, FL, USA. doi: 10.2514/6.2020-0494

Pandit, R. K., \& Infield, D. (2018). SCADA-based wind turbine anomaly detection using Gaussian process models for wind turbine condition monitoring purposes. IET Renewable Power Generation, Vol.12 (11), pages: 1249-1255. doi: 10.1049/iet-rpg.2018.0156.

Reddy, D. M., Kumar, P. S., \& Raju, N. N. (2011). Method and system for monitoring wind turbine, United States Patent, 2011/0142621

Soualhi, A., Yasumine, H., Medjaher, K., Clerc, G., Razik, H., \& Guillet, F. (2018). PHM survey: Implementation of signal processing methods for monitoring bearings and gearboxes. International Journal of Prognostics and Health Management, vol. 9 (2) 28, pages: 14.

Stetco, A., Dinmohammadi, F., Zhao, X., Robu, V., Flynn, D., Barnes, M., Keane, J., \& Nenadic, G. (2019). Machine learning methods for wind turbine condition 
monitoring: A review. Renewable Energy, vol. 133, pages: 620-635. doi: 10.1016/j.renene.2018.10.047

Yang, W., Court, R., and Jiang, J. (2013). Wind turbine condition monitoring by the approach of SCADA data analysis. Renewable Energy, Vol. 53, pages: 365-376. doi: 10.1016/j.renene.2012.11.030

Zhao, F., Tian, Z., and Zeng, Y. (2013). Uncertainty quantification in gear remaining useful life prediction through an integrated prognostics method. IEEE Transactions on reliability, vol. 62 (1), pp. 146-159.

\section{BIOGRAPHIES}

Norio Takeda is a chief researcher at Center for Technical Innovation -Mechanical Engineering in the R\&D group of Hitachi, Ltd. He received the B.S., M.S. and Ph. D. degrees in materials and manufacturing science for Osaka University, Suita, Osaka, Japan, in 1995, 1997, and 1999, respectively. He was also a visiting scholar of Optimal Design (ODE) Laboratory and worked on the optimal design of mechanical structures in the University of Michigan, Ann Arbor, MI, the USA, in 2009-2010. He has focused on the methods of evaluating material fatigue under complex loading conditions and optimizing the design of mechanical structures for 20 years. In addition, he has worked on design and O\&M improvement by utilizing big data collected with the Internet of Things since 2014. He is a member of the Japan society of mechanical engineers (JSME), the society of materials science, Japan (JSMS), and international society for structural and multidisciplinary optimization (ISSMO).

Tatsuya Kameyama is a researcher at Center for Technical Innovation -Artificial Intelligence in the R\&D group of Hitachi, Ltd. He has focused on the methods of big-data analytics and data visualization for 10 years. In addition, he has worked on design and O\&M improvement by utilizing big data collected with the Internet of Things since 2014. He is a member of the Institute of Electronics, Information and Communication Engineers in Japan (IEICE). 